\title{
El medio es el contenido: Comunicación y evaluación del diseño paramétrico en un ambiente MOOC
}

\author{
The medium is the content: Communication and evaluation of parametric design in a MOOC \\ environment.
}

\author{
Rodrigo Shiordia López \\ Universidad Anáhuac México, México \\ rodrigo.shiordia@anahuac.mx
}

\begin{abstract}
This paper describes the implementation of a massive online open course of parametric design. Firstly, the justification for understanding design education in an online system is discussed. A conceptualization of computational geometry as a didactic axis for teaching parametric design is posed. To achieve an evaluation of the course, a peer evaluation which had a rubric for each topic, based on a matrix of possibilities, was implemented. The viability of this evaluation system is discussed. Finally, the important and necessary items for success, as well as the disadvantages of this type of courses is concluded.
\end{abstract}

Keywords: Diseño Paramétrico; Educación en línea; MOOCs; Geometría Computacional

\section{Introducción}

El medio es el mensaje, la frase célebre de Marshall Mcluhan (McLuhan \& McLuhan, 2013) nos habla de cómo el medio en el que se transmite un mensaje es el mensaje mismo. Esto nos obliga a pensar más profundamente en los modos de transmisión de la información, y en sus configuraciones subyacentes.

Hoy en día, la educación del diseño arquitectónico se sostiene primordialmente en la figura del taller. El taller como el espacio de intercambio entre el profesor y el alumno, donde el alumno presenta sus iteraciones de diseño al profesor, y éste crítica y enseña a la vez apuntando errores, marcando directrices, y haciendo preguntas. Este método, al menos en México, ha permanecido desde el siglo XX.

En los últimos años ha habido un cambio de paradigma en el método de representar la arquitectura: los sistemas CAD (Diseño asistido por ordenador, por sus siglas en inglés). Los sistemas de representación, documentación y visualización del diseño arquitectónico han impactado fuertemente a la profesión. Sin embargo, los modelos educativos que surgieron desde metodologías de representación tradicionales han persistido en su formato habitual y poco se ha hecho en términos de exploración de metodologías alternativas.

Al mismo tiempo, existen esfuerzos en donde las técnicas de representación digital van más allá de la documentación del diseño, y se convierten en el motor del diseño mismo. Se han implementado sistemas de programación visual, scripting, y algoritmos que permiten un alto control geométrico y un diseño emergente donde los sistemas formales se diseñan de abajo hacia arriba.

Si el medio es el mensaje, y el medio es el ordenador, ¿no debe de existir un modelo educativo más cercano a esta tecnología? Uno de los sistemas de intercambio de información más universales es el internet. Una de las lógicas del intercambio de información de este sistema, es el cambio en los tiempos de respuesta. En el correo electrónico, la comunicación es distendida, no es inmediata, es en los términos del usuario; lo mismo sucede en un foro o un sitio wiki. (Rushkoff, 2011). Uno deja un mensaje, o deja una publicación en un foro y el destinatario lo lee, hasta que está frente a su pantalla. Es más rápido que el correo, pero más lento que una conversación en tiempo real.

Esta distensión en la temporalidad de la comunicación, cuando es vista en un ámbito educativo, encuentra su referente en los Cursos en Línea Masivos y Abiertos (MOOCs por sus siglas en inglés). Un MOOC es un curso abierto a todo el público, al que cualquiera puede entrar. La información en un MOOC debe ser presentada en un formato que permita ser leída en esta temporalidad distendida que permiten los sistemas informáticos de redes de información.

En este contexto, se propone un modelo educativo del diseño que no sólo se adapte a los medios educativos de vanguardia, sino que interiorice los medios de producción del diseño mismo: Si estamos diseñando usando el ordenador, ¿porqué no podemos aprender a diseñar por completo usando el ordenador? Este trabajo presenta las ideas principales detrás de un curso de diseño paramétrico implementado en un ambiente MOOC.

Este curso se presenta como una alternativa al modelo tradicional de enseñanza del diseño, en donde las nociones básicas como retroalimentación, seguimiento y evaluación se deben de repensar por completo al tratarse de un medio completamente distinto. Es por esto por lo que se plantea este curso como el medio y el contenido al mismo tiempo, el curso enseña material digital, en una plataforma digital, con resultados exclusivamente digitales. Si bien es cierto que este 
esfuerzo dista mucho para sustituir al modelo educativo tradicional, se puede entender también como complemento para la educación de temas específicos en la estructura curricular de un programa educativo de diseño.

Al mismo tiempo, este curso se plantea como una posición crítica acerca de la manera en la que se debe presentar el diseño paramétrico para que el alumno pueda implementar técnicas avanzadas de modelado y se produzcan resultados con alta complejidad informados por datos.

\section{Planteamiento del curso}

\section{Temario}

Para poder enseñar al alumno el concepto de diseño paramétrico, se buscó que el alumno comprendiera los objetos básicos de la geometría computacional. Estos objetos se plantearon como los ejes directores de cada módulo del curso:

\section{Módulo 1}

a. Introducción e instalación

b. Interfaz, componentes y tipos de datos

c. Geometrías básicas: Puntos, planos y vectores

d. Matemáticas, funciones, expresiones y condicionales

2. Módulo 2
a. Geometrías intermedias: Curvas
b. Diseñando con listas
c. Visualización de listas
d. Manejo de listas de datos

3. Módulo 3
a. Geometrías avanzadas: Superficies
b. Geometrías avanzadas: Mallas
c. Árboles y conjuntos
d. Diseñando con árboles

4. Módulo 4
a. Weaverbird: Trabajo con mallas
b. Ladybug: Análisis climático
c. Kangaroo: Simulación de fuerzas
d. Poniéndolo todo en perspectiva

En el módulo 1 se trabajaron los puntos, los planos, y los vectores como objetos geométricos computacionales básicos, se trabajó el concepto de dato, de tipo de dato y de cómo se pueden establecer relaciones entre datos para generar objetivos determinados. El vector, en realidad es una terna de datos, que marca una dirección y una magnitud, y se usa este tipo de dato extensivamente en diseño paramétrico, pues la forma arquitectónica, siempre presenta direccionalidad. Otro concepto importante es entender el espacio como un campo vectorial, lo cual nos permite convertir el espacio en un campo medible de intensidades. (Shiordia López \& Gerber, 2014). En este módulo, se presentó un ejercicio donde se produjo un campo vectorial con puntos atractores, generando así un campo direccional.

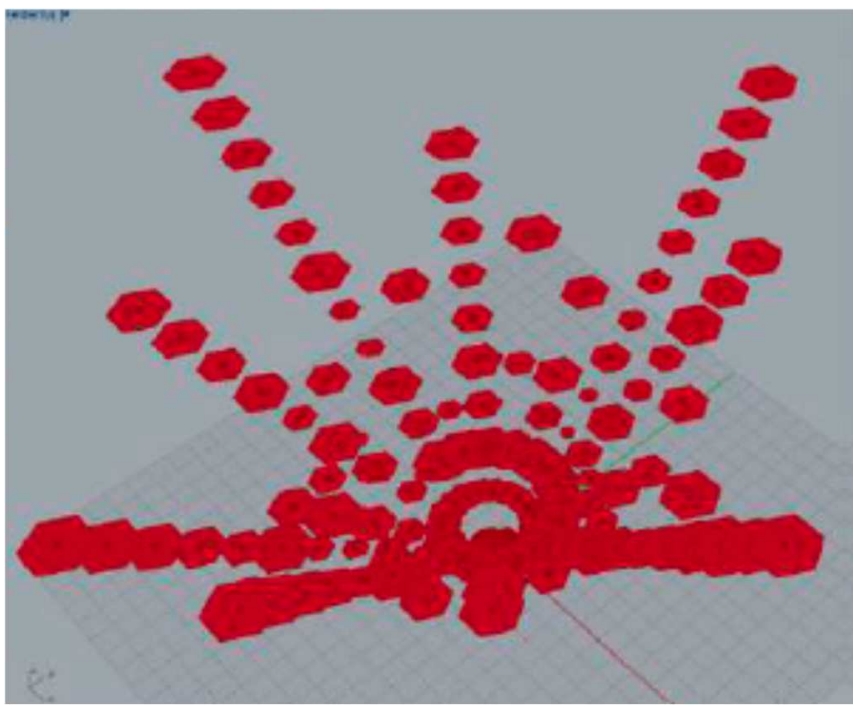

Figura 1: Captura de pantalla de un ejercicio del módulo 1 en el que los alumnos implementaron operaciones con puntos, vectores, y expresiones matemáticas.

El módulo 2 presentó el concepto de curva, no en el sentido literal de una curva como un trazo no-recto, sino en su concepto digital como conjunto de puntos, tangencias, grados y puntos de control. Esto permitió profundizar en el concepto de entrada-salida, tan importante en un sistema computacional. (Pottmann, Asperl, Hofer, \& Kilian, 2007) La Manera de entender una curva como un operador que conjunta una lista de puntos nos permite entender los conceptos computacionales y los geométricos al mismo tiempo. Uno de los ejercicios de este módulo fue la producción de curvas sinoidales, espirales y helicoidales a partir de expresiones matemáticas. La expresión matemática evaluada a partir de una lista de números, generando así una lista de resultados usados como entrada para componentes de puntos que eran los constructores de las curvas.

Las exploraciones acerca de las formas volumétricas se dieron en el módulo 3, en donde se exploraron las superficies y mallas desde sus entradas, es decir, la manera paramétrica de construir estos objetos, a través de operadores como revoluciones, barridos etc. Aquí, se presentó la idea de la superficie como campo, como espacio paramétrico, que puede ser evaluada, dividida y se pueden extraer sus normales y tangentes para construir geometrías sobre ella. El ejercicio final de este módulo fue la generación de una panelización a partir de una superficie de doble curvatura de grado 3. El alumno aprendió a extraer las normales de la superficie, y modificarlas para imprimir direccionalidad a su acomodo.

El módulo 4 buscó extender la funcionalidad hacia herramientas avanzadas de manejo de mallas y de análisis de superficies, así como la simulación de fuerzas. Mientras este módulo no buscaba ser un referente en la simulación computacional, sí buscaba implementar el concepto de simulación como motor del diseño. (Delanda, Philosophy and Simulation: The Emergence of Synthetic Reason, 2011) 
La simulación como motor del diseño trata en este curso de ser aproximada desde el enfoque de los datos, y las relaciones posibles entre estos datos y las geometrías de los alumnos. La simulación puede ser una herramienta muy poderosa cuando es entendida como un campo de información que informa las propiedades o parámetros de una geometría (Gerber, Shiordia López, Veetil, \& Mahesh, 2014). En este caso, se trató de presentar la metodología de dos tipos de simulación principal: simulación de fuerzas, y simulación de radiación solar.

La primera idea de simulación fue la simulación física a través del uso de los componentes de la extensión de Grasshopper llamada Kangaroo. Se establecieron los criterios básicos para poner en marcha una simulación de una red de resortes y relajar los resortes aplicando una simple fuerza unitaria unidireccional. Esta metodología ha sido discutida ampliamente y hay ejemplos construidos a distintos niveles. (Bieg, 2014) Los alumnos implementaron una geometría inicial, para después "relajarla" y encontrar nuevas configuraciones al aplicar distintas fuerzas.

La segunda metodología de simulación presentada fue la simulación de incidencia solar basada en mallas de la extensión Ladybug de Grasshopper. Esta extensión permite disponer una simulación y cálculo preciso de incidencia de radiación solar, que puede ser visualizado o usado como datos y parámetros para enriquecer los modelos de los alumnos.

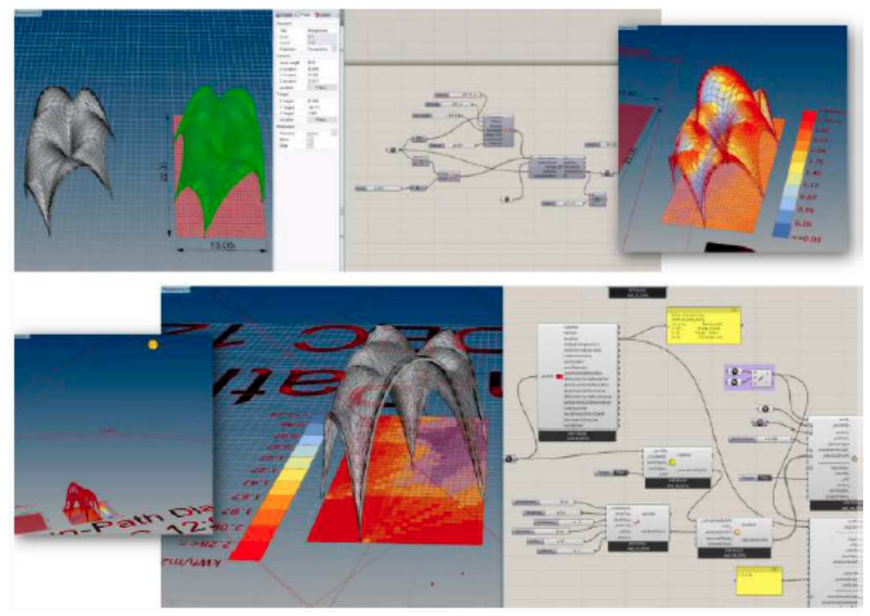

Figura 2: capturas de pantalla del trabajo de un alumno que conjunta las superficies relajadas y un análisis de radiación solar sobre la superficie, y en el suelo debajo de ella.

\section{Conceptualización del Diseño Paramétrico}

En un sistema educativo de composición arquitectónica tradicional, se presentan los componentes básicos de una composición como punto, línea, plano y volumen. (Ching, 2002), en un sistema de diseño paramétrico, el curso busca traducir estos elementos a sus análogos computacionales, elementos que conjuntan información adicional además de su propia espacialidad geométrica. Así, el curso conceptualiza los análogos de la siguiente manera:

El punto como vector. El vector, como hemos dicho antes codifica además de una posición, una direccionalidad, y dentro de este sistema, el vector también puede asumir operaciones geométricas, así el vector puede adicionarse, sustraerse, o multiplicarse con otro vector, cosa que es imposible para un punto. En el diseño paramétrico, estas operaciones son importantes, pues permiten producir operaciones que abren nuevas matrices de posibilidades.

La línea como curva. Hemos discutido la definición de curva en el contexto de geometría computacional. Una línea en el sentido tradicional, siempre se entiende en su definición virtual, sin embargo, en el contexto del diseño paramétrico y la geometría computacional, se entiende la curva desde sus parámetros de entrada: puntos de control, grado, tangencia, etc. Estos parámetros de entrada representan una aproximación más real a la definición matemática de una línea como una sucesión de puntos, y se aleja de la concepción tradicional de línea como eje. Así, la línea se convierte en curva, y a través de la curva, podemos comenzar a entender tanto los operadores básicos del diseño paramétrico, como un nuevo paradigma de entendimiento de la forma. La curva entendida como componente básico de una composición representa una estrategia para generar aberturas, intersecciones, contornos, etc. (Schumacher, 2006)

El plano como superficie. En un sistema tradicional, siempre se entiende el plano como la extensión compositiva de una línea, sin embargo, la superficie, en este curso, se convierte en un objeto resultante de interacciones entre curvas de construcción, puntos de control, etc. La superficie es entendida comoo un campo de referencia de cambios de curvatura. Un espacio de rapideces y lentitudes. (Delanda, Real Virtuality, 2011)

El volumen como sólido, como malla. En cuanto el curso pasa de la conceptualización del plano a la superficie, y transita hacia el concepto de malla, de sólido, se empieza a entender el volumen de manera distinta. En vez de entender el volumen como una composición de planos y caras, se empieza a entender el volumen como la discretización de la forma por medio de caras. Es decir, la malla se convierte en algo con muchas más posibilidades, pues cada cara puede ser accesada en un árbol de datos y se puede operar en y con la cara individual y colectivamente, generando resultados emergentes. Este tipo de resultados sólo son posibles en un contexto de diseño paramétrica, pues la concepción de la forma tradicional no permite entender estas operaciones colectivas.

\section{Evaluación y Comunicación del Diseño}

\section{El modelo paramétrico como resultado del aprendizaje}

Un modelo paramétrico es una representación simbólica entre objetos geométricos que está controlado por valores o 
parámetros. Por ejemplo, una línea en geometría tradicional es la sucesión de puntos infinitamente divisible entre dos puntos. El modelo paramétrico extiende esta definición para representar simbólicamente, que los dos puntos dados, pueden ser cualesquiera puntos dados, y el modelo paramétrico sólo muestra su resultado al recibir una entrada (Input/Output). Así, el modelo paramétrico permite al diseñador producir un número infinito o casi infinito de resultados a través de las relaciones entre objetos. El objetivo del curso era que el alumno pudiera entender este concepto y que interiorizara las técnicas necesarias para producirlo en un ambiente CAD. En este caso se utilizó Rhinoceros 5 y Grasshopper 0.99 .

No se pretende presentar una definición de diseño paramétrico a partir de la crítica o teoría arquitectónica. No se busca entrar en las cuestiones estilísticas que definen al diseño paramétrico. Más bien, se define en este curso el diseño paramétrico como el diseño que parte del modelo paramétrico para generar forma.

\section{La matriz de posibilidades}

El curso estaba dividido en 4 módulos. Cada uno de los tres primeros estaban orientados a un objeto geométrico. El punto/vector, la línea/curva, y el plano/superficie. Al mismo tiempo, el curso fue profundizando poco a poco en conceptos importantes de programación visual, como lo son las listas, las listas anidadas y los tipos de datos. Estos conceptos son ajenos al mundo del diseño, y el curso tomó como postura el explicarlos a través del lenguaje de la forma.

Hemos discutido la definición de modelo paramétrico, sin embargo, al ser este modelo el resultado de aprendizaje esperado se planteó que la manera idónea para presentar los resultados de cada módulo fuera una matriz de posibilidades. En esta matriz, se buscaba que el alumno presentara un espacio de solución dentro de su modelo paramétrico, pues a través de la multiplicidad de posibilidades se podía estudiar diferentes efectos de combinaciones de parámetros. La matriz de posibilidades era entonces el entregable de cada módulo, donde el alumno producía un modelo paramétrico, y presentaba un número de opciones resultantes de las relaciones que él mismo planteó.

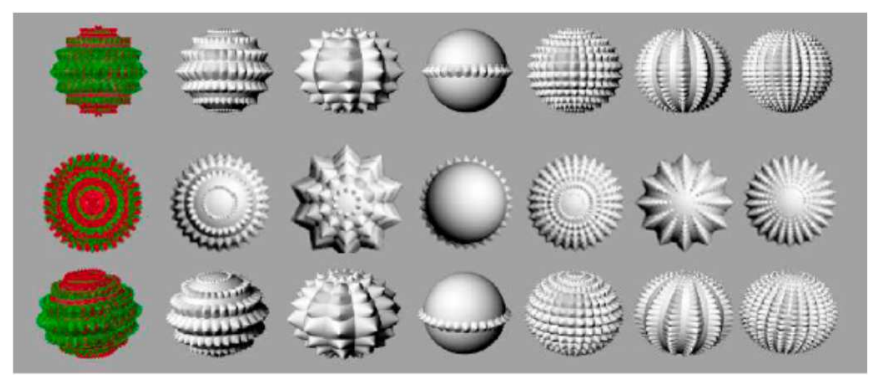

Figura 3: Matriz de Posibilidades entregada por un alumno. Se observan diferentes vistas de diferentes versiones del modelo paramétrico. En este caso es el ejercicio del módulo 2, en el que se usan los conceptos de superficie.

\section{El ambiente MOOC en la enseñanza del diseño paramétrico}

El curso estaba estructurado alrededor de tres ejes principales: Conceptos de geometría computacional, técnicas específicas de una paquetería de software, y el pensamiento paradigmático del diseño paramétrico. La manera en la que se decidió transmitir estos conocimientos era a través de lecturas, videos tutoriales, y archivos descargables. Las lecturas explicaban conceptos básicos que se trataban en los videos. Se produjeron 16 videos tutoriales del proceso para producir un modelo paramétrico en cada uno. En total, fueron cerca de 6 horas de video. Los archivos descargables mostraban el proceso terminado y especificaban los parámetros que el alumno podía modificar. Cada módulo consistía de tres actividades principales, los videos, una lectura, y un ejercicio creativo. Al desarrollar estas tres actividades: el video, la lectura, y la exploración, se plantea que se puede interiorizar el material y las técnicas.

Uno de los retos más importantes de este curso era encontrar una manera de evaluar un diseño de manera objetiva sin contar con la interacción en tiempo real. El diseño es una actividad muy subjetiva, y los resultados del mismo también lo son. Al mismo tiempo, el tema del curso también era altamente técnico y más una habilidad que un conocimiento, por lo cual era difícil encontrar modalidades de evaluación adecuadas. Para esto se plantearon dos evaluaciones para cada módulo. La primera evaluación consistía en un examen de opción múltiple que evaluaba la retención de los conceptos técnicos, la segunda evaluación, era una evaluación entre pares de una matriz de posibilidades del modelo paramétrico. El alumno al enviar su matriz recibía una de parte de otro alumno y con una lista de cotejo o rúbrica, podía evaluar a un compañero. Para estas evaluaciones, se usó una rúbrica, que el alumno conocía de antemano, y con la que evaluaba a su par.

\section{Resultados y Discusión}

El curso, en sus dos ediciones, conto con más de 3,000 inscritos, cerca de 500 lo terminaron y recibieron su certificación, y fue uno de los primeros materiales en línea en español que abordan el tema.

Durante el curso, el papel del profesor fue sólo moderar los foros de discusión y responder las preguntas en la zona delimitada para ello en el sitio. En las preguntas iniciales, el problema más persistente estuvo relacionado a las compatibilidades de los softwares con diferentes sistemas operativos, o dudas de cómo entrar a cierto contenido. Una vez superado esto, las preguntas algunas veces eran resueltas por los mismos alumnos.

Otro tema importante fue la transmisión de la información, los formatos y diferentes tamaños de archivos representaban un obstáculo para las evaluaciones de pares.

El trabajo de preparación para lograr un curso de este tipo es intenso. Se ha discutido con anterioridad la importancia de que el contenido previo esté elevadamente desarrollado (Stellingwerff, 2015). Mientras que el trabajo previo de 
preparación fue intenso, el desarrollo del curso fue satisfactorio, ya que el profesor sólo se dedicó a responder preguntas puntuales en el foro del curso. Los alumnos lograron producir sus propios modelos paramétricos, y presentar sus opciones de diseño en su matriz de posibilidades.

Se han discutido diversos métodos de presentar temas y conocimientos de diseño en ambientes MOOC (Kovács \& Szoboszlai, 2016). Estos esfuerzos muestran cursos con un alto nivel de enseñanza y aprendizaje, sin embargo, no se discute un sistema viable de evaluación, que se encuentre relacionado al material impartido. El sistema descrito en este trabajo, basado en una matriz de posibilidades, una evaluación de pares, y una rúbrica, puede representar un camino satisfactorio para que el alumno interiorice el material, e implemente el diseño paramétrico de manera creativa en poco tiempo. La evaluación de pares obliga al creador de contenido, el profesor, a sintetizar los puntos principales de la evaluación, y el alumno cuenta con estos desde un principio. El alumno evalúa a sus pares de manera horizontal, reduciendo la carga de trabajo del profesor (es un curso masivo) para hacer de la evaluación una actividad del alumno. Un posible peligro de esta evaluación es la diferencia de criterios entre los alumnos, sin embargo, esto se debe de minimizar al implementar rúbricas específicas con criterios sólidamente definidos que permitan a cualquier alumno evaluar a otro.

Una desventaja de este sistema es la manera en que se presenta la información, si no hay un formato establecido para cada entrega, es complicado implementar la evaluación de pares, pues los cambios en formato pueden confundir a los participantes. De ahí la elección de la matriz de posibilidades como punto de partida para la presentación de un espacio de solución de un modelo paramétrico.

En el futuro, para evaluar correctamente el alcance y efectos específicos de este curso, es necesario implementarlo nuevamente con una encuesta específica de entrada, donde se evalúe la experiencia previa con diseño paramétrico de los alumnos. Asimismo, también será necesario evaluar con una encuesta las opiniones de los alumnos, para determinar cambios al material que deban hacerse. Estas encuestas no se implementaron en este curso, pero deberán ser usadas en cursos posteriores.

El éxito de este curso tuvo como insumo indispensable el contenido previo de alta calidad. Los videos, tutoriales y lecturas fueron creados y seleccionados con mucho detenimiento. Asimismo, fue importante presentar los resultados de aprendizaje de una manera adecuada para el contenido. La matriz de posibilidades se convierte en una herramienta instrumental a la hora de presentar las potencialidades de un modelo paramétrico. Por último, la evaluación de pares fue un elemento indispensable para no hacer del curso una carga excesiva para el profesor.

Esta modalidad de educación se posiciona como una alternativa viable para la enseñanza del diseño asistido por computadora y el diseño paramétrico, ya que el medio de trasmisión del conocimiento es el mismo en el que se estás desarrollando el aprendizaje, es decir el alumno aprende en su computadora, a su ritmo y con sus propios horarios. Esta metodología también presenta una alternativa interesante para el profesor como generador de contenido creativo y facilitador del aprendizaje. En una época en la que la profesión del diseño está sufriendo grandes cambios, es importante entender los medios que usamos para transmitir la información, y usarlos en sus propias potencialidades y capacidades. La enseñanza de un nuevo paradigma de diseño requiere de la transmisión de sus conceptos dentro de un nuevo paradigma educativo.
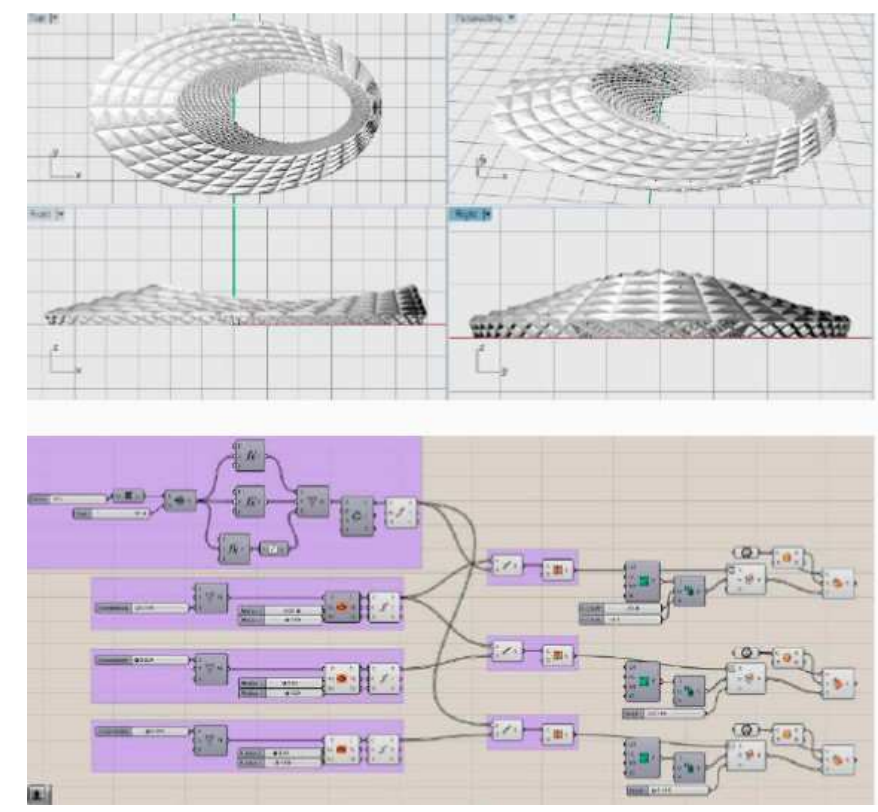

Figura 4: Modelo paramétrico final de una alumno con su programación visual.

\section{Agradecimientos}

Este curso en línea surge como programa piloto para el proyecto MOOC Anáhuac de la Universidad Anáhuac, liderado por la Dra. Diana Galindo Sontheimer de la Dirección de Desarrollo Académico e Investigación. Doy las gracias a dicha instiución y a la Coordinación de Tecnologias para la Educación de la Universidad Anáhuac, sin cuya ayuda no hubiera sido posible. El curso fue posible gracias a Mireya López Acosta, Rosario González Herrera, Guillermo Morfín Morales, Juan Pablo Granados Carmona, Alicia Hernández Barba y sus alumnos. Este trabajo se realizó gracias al apoyo del Dr. Arq. Bernardo Gómez-Pimienta Magar, el Arq. Emilio Castro, y toda la gente en la Escuela de Arquitectura de la Universidad Anáhuac México Norte.

Agradezco también al comité organizador SiGraDi 2017 por considerar este tema de interés en el simposio y por sus comentarios editoriales. 


\section{Referencias}

Bieg, K. (2014). Caret 6 and the Digital Revival of Gothic Vaults. ACADIA 14: Design Agency (Proceedings of the 34th Annual Conference of the Association for Computer Aided Design in Architecture) (pp. 199208). Los Angeles: ACADIA.

Ching, F. (2002). Arquitectura: Forma, Espacio y Orden. Barcelona: Gustavo Gili.

Delanda, M. (2011). Philosophy and Simulation: The Emergence of Synthetic Reason. New York: Continuum.

Delanda, M. (2011). Real Virtuality. In A. Mengues, \& S. Alqhuist, Computational Design Thinking (pp. 142146). London: Wiley.

Gerber, D. J., Shiordia López, R., Veetil, S., \& Mahesh, A. (2014). Design agency: prototyping multi-agent system simulation for design search and exploration. SimAUD '14 Proceedings of the Symposium on Simulation for Architecture \& Urban Design (pp. 160168). Tampa, Florida: SimAUD.

Kovács, Á., \& Szoboszlai, M. (2016). Experience in CAAD Education Using a MOOC System. Proceedings of the 34th eCAADe Conference - Volume 1 (pp. 269274). Oulu, Finlandia: ECAADE.
McLuhan, M., \& McLuhan, M. (2013). Understanding Media: The Extensions of Man. Berkeley: Ginko Press.

Pottmann, H., Asperl, A., Hofer, M., \& Kilian, A. (2007). Architectural Geometry. London: Bentley Architectural Press.

Rushkoff, D. (2011). Program or Be Programmed: Ten Commands for a Digital Age. Berkeley: Soft Skull.

Schumacher, P. (2006). Arguing for Elegance. (A. R. Helen Castle, Ed.) Elegance, $A D$ (Architectural DEsign), $77(1), 28-37$.

Shiordia López, R., \& Gerber, D. (2014, Noviembre 12,13,14). Context-Aware Multi Agent Systems: Negotiating Intensive Fields. (SIGRADI, Ed.) SIGraDi 2014 [Proceedings of the 18th Conference of the Iberoamerican Society of Digital Graphics, 138-143.

Stellingwerff, M. (2015). The MOOC-ability of Design Education. Proceedings of the 33rd eCAADe Conference - Volume 2, Vienna University of Technology (pp. 57-60). Vienna, Austria: ECAADE.

Williams, J. H. (2008). Employee engagement: Improving articipation in safety. . Professional Safety, , 12(53), 40-45. 